\title{
Larix laricina, an Antidiabetic Alternative Treatment from the Cree of Northern Quebec Pharmacopoeia, Decreases Glycemia and Improves Insulin Sensitivity In Vivo
}

\author{
Despina Harbilas, , 2, 3, 4 Diane Vallerand, ${ }^{1,2,3,4}$ Antoine Brault, ${ }^{1,2,3,4}$ Ammar Saleem, ${ }^{1,4,5}$ \\ John T. Arnason, ${ }^{1,4}$ Lina Musallam, ${ }^{1,2,3,4}$ and Pierre S. Haddad, 2, 3,4 \\ ${ }^{1}$ Canadian Institutes of Health Research Team in Aboriginal Antidiabetic Medicines, Department of Pharmacology, \\ University of Montreal, P.O. Box 6128, Downtown Station, Montreal, QC, Canada H3C 3J7 \\ ${ }^{2}$ Natural Health Products and Metabolic Diseases Laboratory, Department of Pharmacology, University of Montreal, \\ Montreal, QC, Canada H3C $3 J 7$ \\ ${ }^{3}$ Institute of Nutraceuticals and Functional Foods, Laval University, Quebec City, QC, Canada G1V OA6 \\ ${ }^{4}$ Montreal Diabetes Research Center, University of Montreal Hospital Center, Montreal, QC, Canada H1W $4 A 4$ \\ ${ }^{5}$ Department of Biology and Center for Research in Biopharmaceuticals and Biotechnology, University of Ottawa, \\ Ottawa, ON, Canada K1N 6N5 \\ Correspondence should be addressed to Lina Musallam, l.musallam@gmail.com \\ and Pierre S. Haddad, pierre.haddad@umontreal.ca
}

Received 10 March 2012; Accepted 7 May 2012

Academic Editor: Vincenzo De Feo

Copyright (๑) 2012 Despina Harbilas et al. This is an open access article distributed under the Creative Commons Attribution License, which permits unrestricted use, distribution, and reproduction in any medium, provided the original work is properly cited.

Larix laricina K. Koch is a medicinal plant belonging to traditional pharmacopoeia of the Cree of Eeyou Istchee (Eastern James Bay area of Canada). In vitro screening studies revealed that, like metformin and rosiglitazone, it increases glucose uptake and adipogenesis, activates AMPK, and uncouples mitochondrial function. The objective of this study was to evaluate the antidiabetic and antiobesity potential of L. laricina in diet-induced obese (DIO) C57BL/6 mice. Mice were subjected for eight or sixteen weeks to a high fat diet (HFD) or HFD to which L. laricina was incorporated at 125 and $250 \mathrm{mg} / \mathrm{kg}$ either at onset (prevention study) or in the last 8 of the 16 weeks of administration of the HFD (treatment study). L. laricina effectively decreased glycemia levels, improved insulin resistance, and slightly decreased abdominal fat pad and body weights. This occurred in conjunction with increased energy expenditure as demonstrated by elevated skin temperature in the prevention study and improved mitochondrial function and ATP synthesis in the treatment protocol. L. laricina is thus a promising alternative and complementary therapeutic approach for the treatment and care of obesity and diabetes among the Cree.

\section{Introduction}

The prevalence of obesity and type 2 diabetes (T2D) has reached epidemic proportions worldwide. In Canada alone, obesity and T2D affect 25\% [1-3] and roughly $6 \%[4]$ of the population, respectively. The incidence of these diseases is worsened among the aboriginal population of Canada. The Cree of Eeyou Istchee (CEI) of the Northern James Bay Area of Quebec are particularly affected where $21 \%$ of adults over the age of 20 are diagnosed as diabetic and $38 \%$ as obese [5-10]. This increased prevalence might be caused by their adoption of more westernized way of living: sedentary lifestyle and nontraditional diet (with increased consumption of carbohydrates and saturated fats) in addition to a low-compliance to modern T2D therapies [11-13]. The implementation of educational programs on lifestyle intervention (diet and exercise) has come to no avail, wherefrom the importance to identify alternative and culturally adapted treatments or solutions for obesity and T2D $[6,9-11,13,14]$.

Currently, around $70-95 \%$ of the population in the world relies on alternative and complementary medicine 
in order to respond to their primary healthcare needs. Since the CEI possess a rich traditional pharmacopoeia, we conducted an ethnobotanical survey to identify plant species with potential to treat symptoms related to T2D [15]. These plant species were screened for their antidiabetic potential in extensive in vitro studies [16-24]. Of the 17 plants identified, Larix laricina $\mathrm{K}$. Koch, belonging to the Pinaceae family, demonstrated antidiabetic potential by increasing glucose uptake and phosphorylation levels of AMPK and ACC in C2C12 myotubes to levels almost comparable to those of metformin $[20,24]$. It also potentiated adipogenesis in 3T3-L1 adipocytes, thus acting like the commonly used thiazolidinedione (TZD), rosiglitazone [24]. In addition, we showed that $L$. laricina was one of the strongest uncouplers by severely disrupting mitochondrial function and decreasing ATP production [20]. Uncouplers increase metabolic rate and therefore fuel consumption in order to compensate for decreased ATP, which makes them potential antiobesity agents [20]. Obesity contributes to $55 \%$ of all cases of T2D, therefore affecting one or the other might have therapeutic potential for both diseases [25].

There are a variety of medications available on the market, which help in the management of T2D. Some of them, such as the antidiabetic medications metformin (biguanide) and exenatide (GLP-1 analogue), have been reported to produce a minimal amount of weight loss, albeit not strong enough to be considered as an antiobesity agent $[26,27]$. The diet-induced obese (DIO) mice are an excellent model to study the well-documented relation of losing weight and improving insulin resistance. It relies on high-calorie diet and inactivity (no genetic involvement) to induce significant weight gain, hyperglycemia, and hyperinsulinemia, thus reflecting the establishment of the metabolic syndrome and a prediabetic state. Therefore, we wanted to study the antidiabetic and antiobesity potential of L. laricina in the DIO mouse model using two different administration regiments. Animals either received the plant concomitantly with high fat diet (prevention study) or received the plant after becoming obese and pre-diabetic (treatment study).

\section{Materials and Methods}

2.1. Plant Extracts. Specimens of the plant species used in this study, L. laricina K. Koch, of the Pinaceae family, were collected in 2004 from the territories of the CEI of Northern Quebec, Canada. Dr. Alain Cuerrier, taxonomist at the Montreal Botanical Garden, confirmed the botanical identity of the plant species. Voucher specimens of the plant species were deposited in the Marie-Victorin Herbarium of the Montreal Botanical Garden in Montreal, QC, Canada (Whap04-11, Mis03-12, Mis03-47). Crude 80\% ethanolic extract of L. laricina was prepared as previously described [24].

2.2. Animals. Four-week-old male nondiabetic C57BL/6 mice were purchased from Charles River Laboratories (SaintConstant, QC, Canada). All mice had ad libitum access to food and water. They were housed in individual cages and maintained on a $12 \mathrm{~h}$ light-dark cycle in a temperaturecontrolled animal room. All experimental protocols were approved by the animal experimentation ethics committee of the Université de Montréal and were carried out in full respect of the guidelines from the Canadian Council for the Care and Protection of Animals.

2.3. Prevention Protocol. Following acclimatization, the fourweek-old male nondiabetic C57BL/6 mice were divided into four groups of approximately 12 mice each, where they were monitored for 8 weeks. The control groups consisted in administering to one group (Chow) a standard diet purchased from Charles River (18\% protein content, $4.5 \%$ crude fat, Charles River Animal rodent diet) and to another group (DIO) a high-fat diet (HFD) acquired from Bio-Serv (BioServ Diet no. F3282, Frenchtown, NJ, 60\% fat by energy). The remaining groups received the HFD to which was incorporated the dried $80 \%$ crude ethanolic plant extract of L. laricina at levels adjusted to deliver $125 \mathrm{or} 250 \mathrm{mg} / \mathrm{kg}$ body weight.

2.4. Treatment Protocol. Following acclimatization, the fourweek-old male nondiabetic C57BL/6 mice were divided into four groups of approximately 12 mice each. Chow controls received a standard diet (18\% protein content, $4.5 \%$ crude fat; Charles River Animal rodent diet) for 16 weeks. Other groups were fed a HFD (Bio-Serv Diet no. F3282; 60\% energy from fat) for 8 weeks. L. laricina at 125 or $250 \mathrm{mg} / \mathrm{kg}$ was incorporated in the HFD and treatments continued for an additional 8 weeks, with DIO controls receiving only HFD. Based on published observations and criteria, the animals fed a HFD were segregated into low responders (LRs) and high responders (HRs) (roughly 50/50) according to the data just prior to plant administration (at 8 weeks). Indeed, it has been reported that pooling animals with a more normal metabolic profile even when fed with HFD (such as low weight gain, weak IR, and near-normal glycemia; LR) with animals displaying overt obesity and insulin-resistant state when fed HFD (such as high weight gain, frank IR, and hyperglycemia; HR) can yield misleading results [28]. For simplicity reasons, LR fed with the HFD in the presence or absence of the plant extract will not be depicted since they portrayed an almost normal metabolic profile.

The data representing the effect of a HFD on C57BL/6 mice as compared to their $\mathrm{CHOW}$-fed congeners will not be discussed since the DIO mouse model is well established. However, our model (in both the prevention and treatment studies) follows the expected and published data. In addition, the CHOW group was used as a nonobese control to insure that the model is functional, and therefore the results of this group are not presented. Therefore, the effects of the plant extract, L. laricina, will be compared to their respective HFD controls for all the stated parameters, for both the prevention and treatment protocols.

2.5. Continuous Physiological and Morphological Parameters. In both protocols body weight, food, and water intake, as well as glycemia, were measured 3 times/week during the course 
of the study, consistently at the same time, day, in the same order by the same person, throughout the entire duration of the protocols. In order to assess blood glucose levels, blood was collected from puncturing the tail vein and measured using a commercial glucometer (Accu-Check Roche, Montreal, QC, Canada). The area under the curve (AUC) was calculated for these parameters, and the total AUC was then separated into two parts: fraction 1 (F1), representing AUC between weeks 0 and 4 and fraction 2 (F2) corresponding to the AUC between weeks 4 and 8 of plant extract administration. This segregation served to determine the temporal course of action of L. laricina in both the prevention and treatment protocols, that is, whether it was effective early in onset (first 4 weeks), later (last 4 weeks), or throughout the study.

2.6. Surgical Procedure. At the end of each experimental protocol, the mice were anesthetized using $50 \mathrm{mg} / \mathrm{kg}$ pentobarbital intraperitoneally and then sacrificed by exsanguination via the inferior vena cava. During the sacrifice, various organs were removed, collected, and weighed, notably, liver, muscle, white adipose tissue (WAT; epididymal and retroperitoneal fat pads), and subscapular brown fat (BAT). All were placed in liquid nitrogen and then stored at $-80^{\circ} \mathrm{C}$ until further use. As for the livers, they were flushed with a physiological saline solution, weighed, and the median lobes were then dissected, immediately placed in liquid nitrogen, and then stored at $-80^{\circ} \mathrm{C}$ until further use.

2.7. Blood Parameters. In order to ensure uninterrupted delivery of plant extract and to avoid the complications of interrupting the dietary plant treatment (for example, drop in food intake and body weight caused by fasting the animals), glycemia, insulin, and adipokines correspond to nonfasting measurements. Plasma insulin, adiponectin, and leptin were assessed by radioimmunoassay (RIA: Linco Research, St. Charles, MO, USA) according to manufacturer's instructions.

2.8. Tissue Triglyceride Measurement. Tissue triglyceride content was measured by grinding up into powder, under liquid nitrogen, around $100 \mathrm{mg}$ of each of the collected liver and muscle samples, and then using Folch's chloroform/methanol $(2: 1)$ extraction method [29]. Triglyceride content was then quantified using a commercial kit (Randox Laboratories Ltd., UK).

2.9. Skin Temperature. In the prevention protocol, after 4 and 8 weeks of treatment, the temperature of the animals was read and recorded with a digital thermometer (ColeParmer Instrument Company, USA) by placing a probe on the external intercostal region of the animal for 2 minutes. This procedure is noninvasive and the least stressful for the animals [30].

2.10. Isolation of Mitochondria and Measurement of Respiration. Following anesthetization, the livers of mice from the treatment protocol were flushed with the Krebs-Henseleit buffer $\left(\mathrm{pH} 7.4,22^{\circ} \mathrm{C}\right)$. Mitochondria were then isolated following the method of Johnson and Lardy, as previously described [31-33].

2.11. Statistical Analysis. Data were analyzed by one-way analysis of variance (ANOVA), followed by post hoc analysis (Bonferroni-Dunn test or Holm-Sidak) as appropriate using Sigma Stat software (Jandel Scientific, San Rafael, CA, USA). Areas under the curve (AUC) were calculated by using PRISM software (GraphPad, San Diego, CA, USA). Data are expressed as mean \pm SEM of the indicated number of determinations. Statistical significance was set at $P<0.05$.

\section{Results}

3.1. L. laricina Significantly Improved Glycemia in the Treatment Protocol. Glycemia levels, which increased with HFD as compared to CHOW (by 19\%; data not shown), were not significantly altered when L. laricina was added concomitantly with the HFD (prevention study; NS; Figures 1(a)-1(c)). However, HFD-induced hyperglycemia (32\% as compared to CHOW congeners; data not shown) significantly decreased in the group receiving the plant as treatment (following 8 weeks on HFD; Figures 1(a)-1(c)). Indeed, blood glucose levels, as measured by the area under the curve (AUC) of glycemia versus time, decreased in a dose-dependent manner by $10 \%$ and $12 \%$ with $125 \mathrm{mg} / \mathrm{kg}$ and $250 \mathrm{mg} / \mathrm{kg}$ doses, respectively ( $\mathrm{AUC}_{\mathrm{T}} ; P<0.05$; Figure $1(\mathrm{a})$ ). In order to determine the temporal aspect of this antihyperglycemic effect, we fractionated the AUC between the first month of treatment (weeks 0-4: AUC $\mathrm{F}_{\mathrm{F}}$; Figure 1(b)) and second month (weeks 4-8: $\mathrm{AUC}_{\mathrm{F} 2} ;$ Figure 1(c)). Our findings show that L. laricina lowered glycemia levels from the onset of the treatment (by $11-13 \% ; P<0.05$; Figure $1(\mathrm{~b})$ ), and this was maintained throughout the treatment, albeit remained significant with highest dose only ( $13 \%$ at $250 \mathrm{mg} / \mathrm{kg} ; P<0.05$; Figure 1 (c)).

3.2. L. laricina Significantly Decreased Insulin Levels in the Treatment Protocol Only, While Decreasing Leptin/Adiponectin Ratio in Both Protocols. Administration of either dose of L. laricina tended to lower insulin levels by $25 \%$ to $35 \%$ in the prevention study, but failed to reach statistical significance (NS; Table 1). In the treatment protocol, however, L. laricina-induced decrease of insulinemia levels reached $72 \%$ with the $250 \mathrm{mg} / \mathrm{kg}$ dose $(P<0.05$; Table 1$)$, suggesting improvement of insulin resistance state and coinciding with the plant's highest effect on glycemia as mentioned above.

Other indicators were measured to confirm the reestablishment of insulin sensitivity. Adipose tissue is considered an endocrine organ, releasing into circulation adipokines, such as leptin and adiponectin, involved in the development of insulin resistance. A decrease of leptin/adiponectin ratio is thus considered as a marker of improved insulin sensitivity. In both prevention and treatment protocols, L. laricina tended to increase adiponectin levels by $16-26 \%$, however, reaching statistical significance with the $125 \mathrm{mg} / \mathrm{kg}$ dose in the prevention protocol only $(P<$ 0.05 ; Table 1$)$. In parallel, leptin levels were reduced with 
TABLE 1: Effects of HFD and L. laricina administration on systemic parameters at sacrifice.

\begin{tabular}{|c|c|c|c|c|c|c|}
\hline & \multicolumn{3}{|c|}{ Prevention protocol } & \multicolumn{3}{|c|}{ Treatment protocol } \\
\hline & $\mathrm{DIO}$ & $\begin{array}{l}\text { L. laricina } \\
125 \mathrm{mg} / \mathrm{kg}\end{array}$ & $\begin{array}{l}\text { L. laricina } \\
250 \mathrm{mg} / \mathrm{kg}\end{array}$ & DIO & $\begin{array}{l}\text { L. laricina } \\
125 \mathrm{mg} / \mathrm{kg}\end{array}$ & $\begin{array}{l}\text { L. laricina } \\
250 \mathrm{mg} / \mathrm{kg}\end{array}$ \\
\hline Insulin (ng/mL) & $9.2 \pm 1.8$ & $6.9 \pm 1.1$ & $6.0 \pm 0.9$ & $39.9 \pm 5.8$ & $29.4 \pm 9.1$ & $11.0 \pm 1.9^{*}$ \\
\hline Leptin $(\mathrm{ng} / \mathrm{mL})$ & $39.3 \pm 3.5$ & $37.7 \pm 3.2$ & $33.1 \pm 2.0$ & $39.0 \pm 3.0$ & $27.1 \pm 5.4$ & $30.9 \pm 2.1$ \\
\hline Adiponectin $(\mu \mathrm{g} / \mathrm{mL})$ & $8.8 \pm 0.7$ & $11.1 \pm 0.8^{*}$ & $10.8 \pm 0.4$ & $11.1 \pm 0.6$ & $10.9 \pm 0.7$ & $12.8 \pm 0.6$ \\
\hline Leptin/adiponectin ratio & $4.9 \pm 0.6$ & $3.7 \pm 0.5$ & $3.1 \pm 0.2^{*}$ & $3.5 \pm 0.2$ & $2.5 \pm 0.5^{*}$ & $2.4 \pm 0.2^{*}$ \\
\hline
\end{tabular}

Measurements were obtained after 8 weeks (prevention) or 16 weeks (treatment) of administration with either HFD (DIO) or L. laricina at 125 or 250 mg/kg, which was incorporated in the HFD for 8 weeks in the prevention protocol and for the last 8 of 16 weeks in the treatment protocol. All values represent the mean \pm SEM (prevention protocol DIO = 11; L. laricina $125=13$; L. laricina $250=13$, and for the treatment protocol DIO = 7; L. laricina $125=5 ;$. laricina $250=8$ ). * denotes that treated groups are significantly different as compared to DIO (one-way ANOVA; post hoc analysis Holm-Sidak or Bonferroni-Dunn test; $P<0.05)$.

L. laricina administration by $4-16 \%$ in the prevention study and by $21-30 \%$ in the treatment study, without, however, being statistically significant (NS; Table 1). Overall, these changes in adipokines levels resulted in significant decrease of the leptin/adiponectin ratio. Indeed, administration of $L$. laricina significantly lowered this ratio by $37 \%$ at $250 \mathrm{mg} / \mathrm{kg}$ in the prevention study $(P<0.05$; Table 1$)$ and by $29-$ $31 \%$ with both doses in the treatment study $(P<0.05$; Table 1). Therefore, L. laricina seems to decrease systemic insulin resistance.

Furthermore, since accumulation of lipids in the liver and skeletal muscle have been implicated in insulin resistance, we measured hepatic and muscular triglyceride (TG) levels. Despite L. laricina decreasing systemic insulin resistance, as suggested by improvement in the aforementioned parameters, hepatic or muscular triglyceride levels were not significantly altered (NS, Table 2).

\subsection{L. laricina Diminished Body Weight Gain in Both the} Prevention and Treatment Protocols, While Decreasing Fat Pad Weight in the Prevention Protocol Only. Continuous measurements of cumulative change in body weight (CCBW), represented as the area under the curve (AUC), revealed that while the effect of L. laricina on body weight gain was immediate in the prevention protocol for both doses $(10 \%$ for $\mathrm{AUC}_{\mathrm{F} 1}$; NS; Figure 2(b)), it only reached significant proportions in the second half of the protocol $\left(\mathrm{AUC}_{\mathrm{F} 2}\right)$ with the highest dose, decreasing it by $14 \%$ as compared to DIO controls $\left(\mathrm{AUC}_{\mathrm{F} 2} ; P<0.05\right.$; Figure $\left.2(\mathrm{c})\right)$. In contrast, in the treatment protocol, L. laricina produced its strongest and most significant effect in the first half of administration lowering $\mathrm{AUC}_{\mathrm{F} 1}-\mathrm{CCBW}$ by $10 \%$ at $250 \mathrm{mg} / \mathrm{kg}$ as compared to $\mathrm{DIO}$ congeners $\left(\mathrm{AUC}_{\mathrm{F} 1} ; P<0.05\right.$; Figure $\left.2(\mathrm{~b})\right)$. However, its antiobesity effect was not maintained; L. laricina reduced $\mathrm{AUC}_{\mathrm{F} 2}$ by only $4 \%$ at $250 \mathrm{mg} / \mathrm{kg}$ (NS; Figure 2(c)).

Consistent with the observed decrease in body weight gain, L. laricina significantly lowered retroperitoneal/abdominal fat pad weight in the prevention study by $15 \%$ at $250 \mathrm{mg} / \mathrm{kg}(P<0.05$; Table 3$)$ as compared to DIO controls. As for the treatment, it corresponded to a slight decrease with both doses (5\%-11\%; NS; Table 3).

It is interesting to note that, in both protocols, mice administered L. laricina maintained similar food intake to their DIO controls (NS; Figures 2(d)-2(f)), while being less prone to gaining weight. Finally, L. laricina exhibited no toxicity as demonstrated by unaltered blood biochemical parameters and tissue histological examination (data not shown).

3.4. L. laricina Improved Mitochondrial Function. Regulation of body temperature requires regulating both heat production and heat loss. Mitochondria metabolism is an important source of heat production. For the most part, variations in the rate of electron transport are directly related to the demand by the cells for ATP. However, exogenous substances, which uncouple mitochondria, can lead to the disruption of oxidative phosphorylation, decreasing ATP synthesis and increasing heat production. Previous screening studies have shown that L. laricina uncoupled mitochondrial function in isolated Wistar rat hepatocytes [20]. Therefore, we used skin temperature as an indirect measure of energy expenditure and possible mitochondrial uncoupling in the prevention study. After 4 and 8 weeks of treatment, we observed a gradual and dose-dependent increase in skin temperature with $L$. laricina administration $(P<0.05$; Figures 3(a) and 3(b)).

Having perfected the isolation of mitochondria in mice, we opted to directly evaluate mitochondrial function in the treatment study in plant-treated mice compared to DIO controls. As expected DIO mice, which have increased fatty acid deposition in the liver, exhibited a lower respiratory control ratio (RCR) accompanied by decreased ATP production, in comparison to CHOW animals, although data variability precluded statistical significance (Table 4). Despite a small sample, animals treated with $250 \mathrm{mg} / \mathrm{kg}$ of $L$. laricina seemed to restore mitochondrial function and capacity to the level of chow values, as observed by an increase in RCR and ATP synthesis (Table 4).

\section{Discussion}

According to the International Diabetes Federation latest figures, the number of people living with diabetes will rise from 366 million in 2011 to 552 millions by 2030 [34]. The magnitude and impact of this disease dictate the urgent need for action. Although several drugs exist on the market to treat diabetes, the need to discover novel therapeutic options is warranted, especially in aboriginal context, such as the CEI $[6,9-11,13,14]$. Indeed, recent lifestyle changes and 


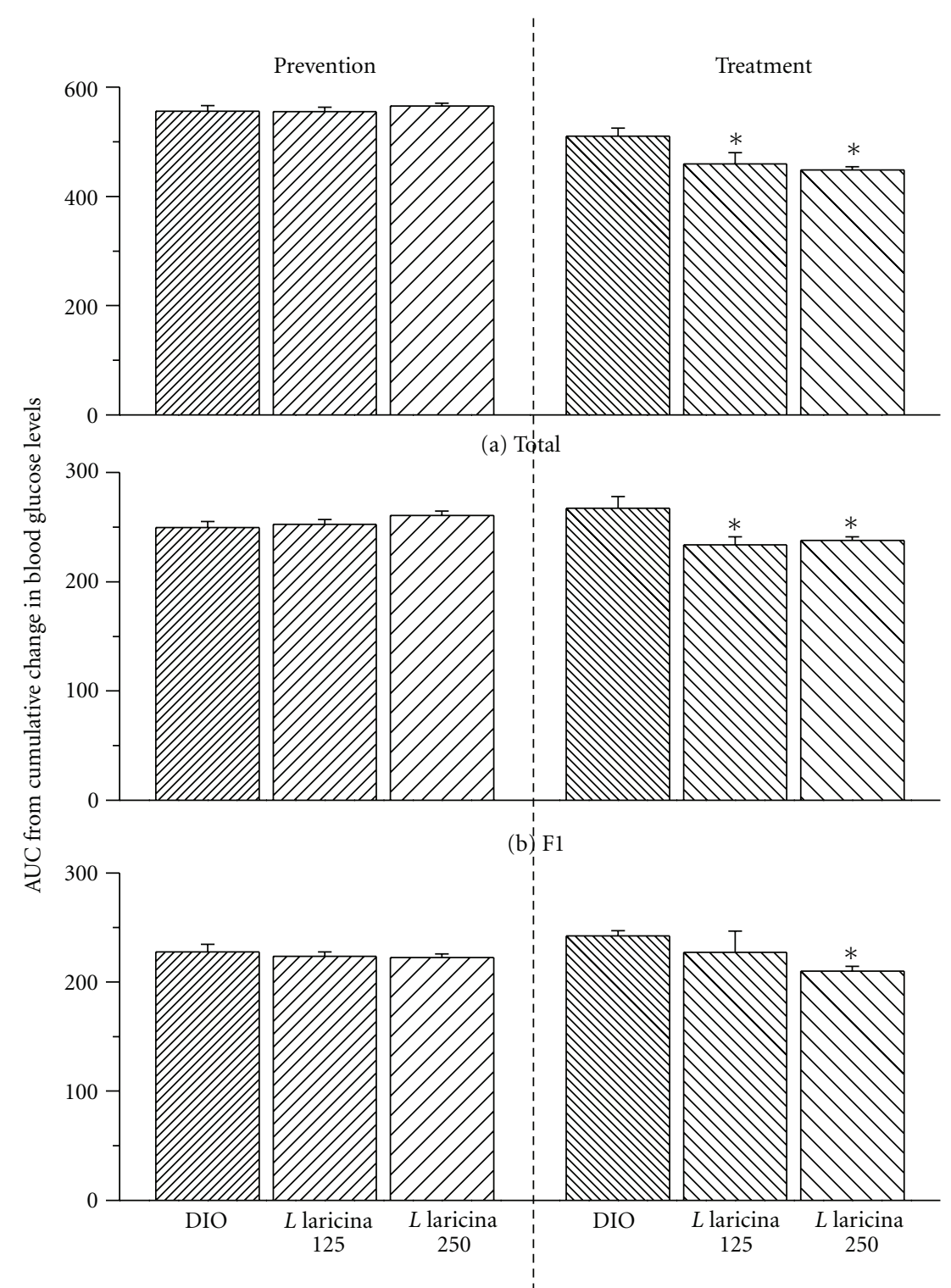

(c) $\mathrm{F} 2$

FIgURE 1: Area under the curve (AUC) of nonfasting glycemia levels, in C57BL/6 mice treated with either HFD (DIO) or L. laricina at 125 or $250 \mathrm{mg} / \mathrm{kg}$, which was incorporated in the HFD for 8 weeks in the prevention protocol and for the last 8 of 16 weeks in the treatment protocol. Total AUC for blood glucose levels versus time (a) was calculated and then fractionated into the first and second half of the feeding period corresponding to weeks $0-4\left(\mathrm{AUC}_{\mathrm{F} 1} ; \mathrm{b}\right)$ and weeks $4-8\left(\mathrm{AUC}_{\mathrm{F} 2} ; \mathrm{c}\right)$, respectively. All values are mean $\pm \mathrm{SEM}$. Number of animals/group for prevention protocol DIO $=11$; L. laricina $125=13$; L. laricina $250=13$, and for the treatment protocol DIO $=7 ;$ L. laricina $125=5 ;$ L. laricina $250=8$ ). * denotes significantly different as compared to DIO group (one-way ANOVA; post hoc analysis Holm-Sidak or Bonferroni-Dunn test; $P<0.05)$.

TABLE 2: Effects of HFD and L. laricina administration on hepatic and muscular triglyceride accumulation.

\begin{tabular}{lccccc}
\hline & & Prevention protocol & \multicolumn{2}{c}{ Treatment protocol } \\
& DIO & L. laricina & L. laricina & DIO & $\begin{array}{c}\text { L. laricina } \\
125 \mathrm{mg} / \mathrm{kg}\end{array}$ \\
& & $125 \mathrm{mg} / \mathrm{kg}$ & $250 \mathrm{mg} / \mathrm{kg}$ & $250 \mathrm{mg} / \mathrm{kg}$ \\
\hline Liver TG levels $(\mathrm{mg} / \mathrm{g}$ total liver) & $331 \pm 54$ & $407 \pm 47$ & $374 \pm 52$ & $1041 \pm 173$ & $919 \pm 240$ \\
Muscle TG levels $(\mu \mathrm{g} / \mathrm{mg})$ & $84 \pm 12$ & $60 \pm 6$ & $65 \pm 8$ & $212 \pm 29$ & $224 \pm 80$ \\
\hline
\end{tabular}

The colorimetric dosage of TG levels in both the liver and muscle was determined using a commercial kit (as described in detail in Section 2.8). Measurements were obtained after 8 (prevention) or 16 (treatment) weeks of administration with either HFD (DIO) or L. laricina at $125 \mathrm{or} 250 \mathrm{mg} / \mathrm{kg}$, which was incorporated in the HFD for 8 weeks in the prevention protocol and for the last 8 of 16 weeks in the treatment protocol. All values represent the mean \pm SEM ( prevention protocol DIO = 11; L. laricina $125=13$; L. laricina $250=13$, and for the treatment protocol DIO = 7; L. laricina $125=5 ;$ L. laricina $250=8$ ). 

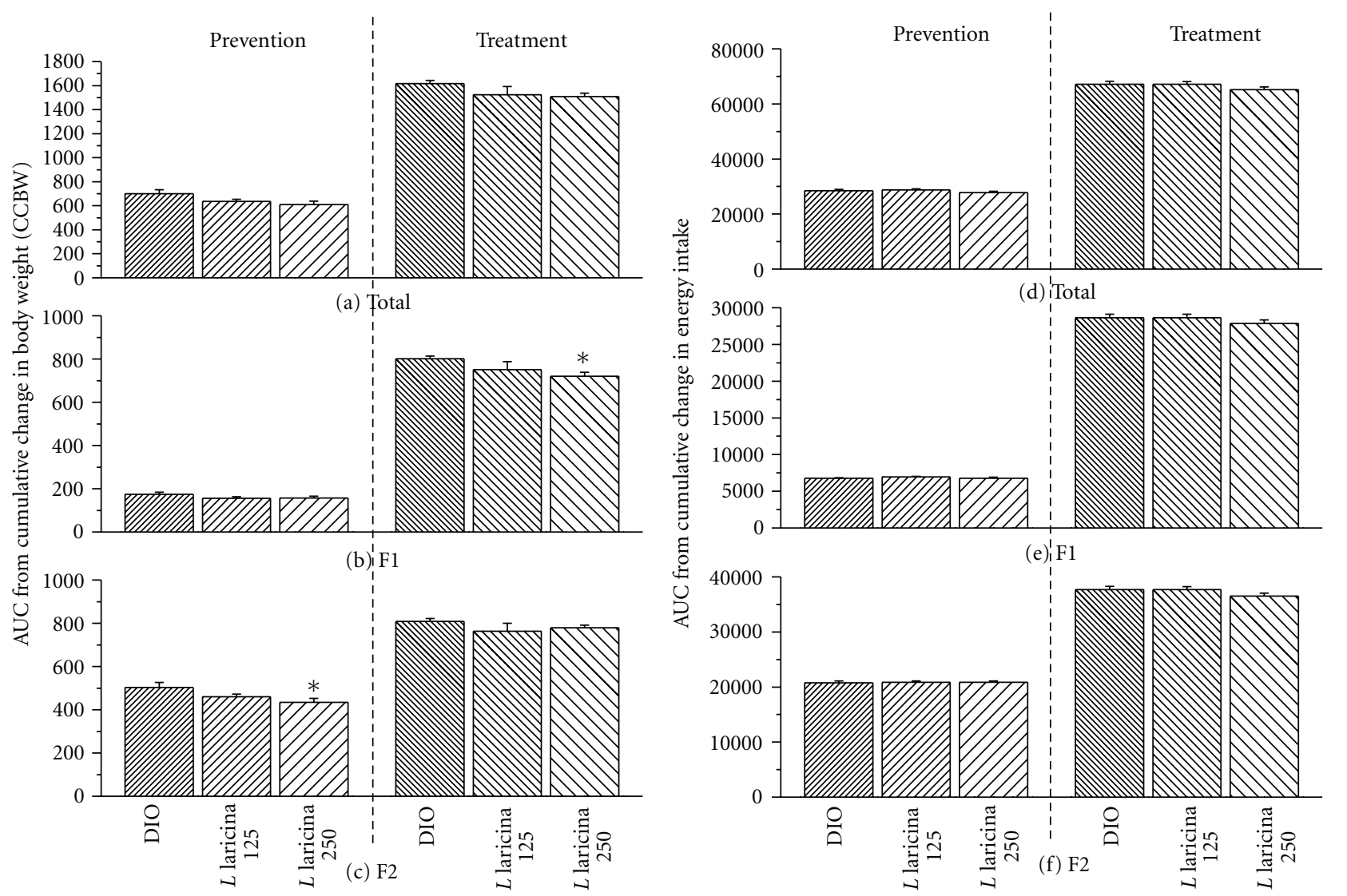

FIGURE 2: Area under the curve (AUC) of cumulative change in body weight (CCBW; $(\mathrm{a}-\mathrm{c})$ ) and cumulative change in energy intake (CCEI; $\mathrm{d}-\mathrm{f}$ ) in C57BL/6 mice treated with either HFD (DIO), or L. laricina at 125 or $250 \mathrm{mg} / \mathrm{kg}$, which was incorporated in the HFD for 8 weeks in the prevention protocol and for the last 8 of 16 weeks in the treatment protocol. Total AUC for CCBW-versus-time (a) or CCEI-versus-time (d) was calculated and then fractionated into the first and second half of the feeding period corresponding to weeks $0-4$ (AUC $\mathrm{F}_{1}$; b or e) and weeks 4-8 (AUC $\mathrm{F}_{2} ; \mathrm{c}$ or $\left.\mathrm{f}\right)$, respectively. All values are mean \pm SEM. Number of animals/group for prevention protocol DIO = 11; L. laricina $125=13$; L. laricina $250=13$, and for the treatment protocol DIO $=7$; L. laricina $125=5$; L. laricina $250=8$ ). ${ }^{*}$ denotes significantly different as compared to DIO group (one way ANOVA; post-hoc analysis Holm-Sidak or Bonferroni-Dunn test; $P<0.05$ ).

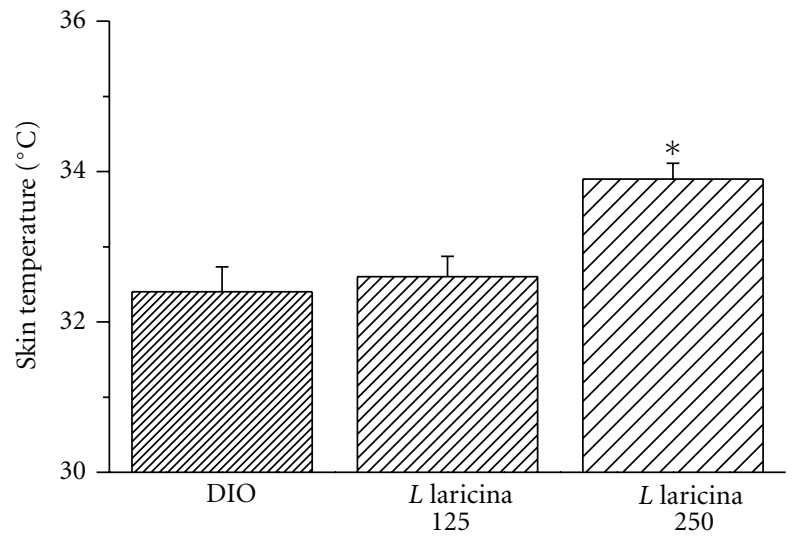

(a)

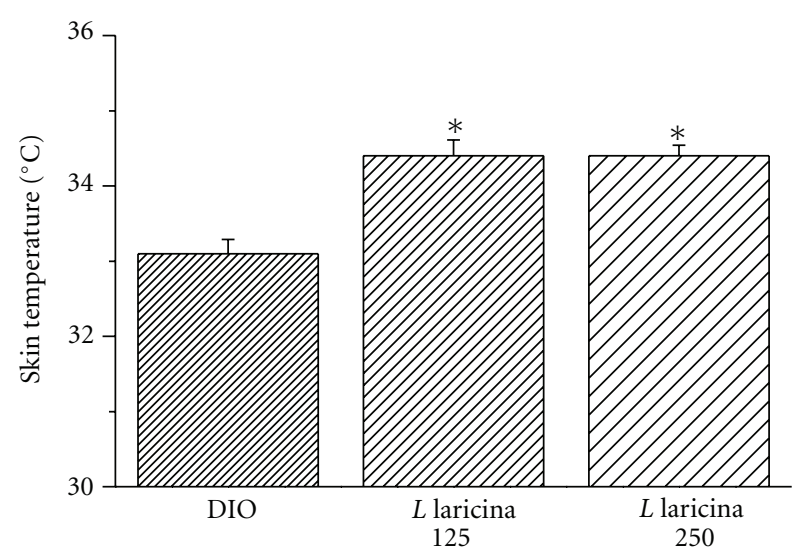

(b)

FIGURE 3: Skin temperature was measured in the prevention study after 4 weeks (a) and after 8 weeks (b) of treatment, from mice treated with either HFD (DIO) or L. laricina at 125 or $250 \mathrm{mg} / \mathrm{kg}$, which was incorporated in the HFD for 8 weeks. All values are mean \pm SEM. Number of animals/group for prevention protocol DIO $=11$; L. laricina $125=13$; L. laricina $250=13$. ${ }^{*}$ denotes significantly different as compared to DIO group (one-way ANOVA; post hoc analysis Holm-Sidak or Bonferroni-Dunn test; $P<0.05$ ). 
TABLE 3: Effects of HFD and L. laricina administration on organ weights at sacrifice.

\begin{tabular}{|c|c|c|c|c|c|c|}
\hline & \multicolumn{3}{|c|}{ Prevention protocol } & \multicolumn{3}{|c|}{ Treatment protocol } \\
\hline & \multirow{2}{*}{$\mathrm{DIO}$} & L. laricina & L. laricina & \multirow{2}{*}{ DIO } & L. laricina & L. laricina \\
\hline & & $125 \mathrm{mg} / \mathrm{kg}$ & $250 \mathrm{mg} / \mathrm{kg}$ & & $125 \mathrm{mg} / \mathrm{kg}$ & $250 \mathrm{mg} / \mathrm{kg}$ \\
\hline Retroperitoneal fat pad (g) & $1.34 \pm 0.05$ & $1.26 \pm 0.04$ & $1.14 \pm 0.05^{*}$ & $1.51 \pm 0.08$ & $1.35 \pm 0.24$ & $1.44 \pm 0.04$ \\
\hline Epididymal fat pad $(\mathrm{g})$ & $2.40 \pm 1.00$ & $2.56 \pm 0.08$ & $2.56 \pm 0.07$ & $1.21 \pm 0.03$ & $1.32 \pm 0.18$ & $1.85 \pm 0.12^{*}$ \\
\hline Brown fat pad (g) & $0.30 \pm 0.03$ & $0.33 \pm 0.01$ & $0.28 \pm 0.02$ & $0.44 \pm 0.01$ & $0.37 \pm 0.06$ & $0.42 \pm 0.02$ \\
\hline Liver weight (g) & $1.77 \pm 0.09$ & $1.85 \pm 0.05$ & $1.80 \pm 0.07$ & $2.62 \pm 0.15$ & $2.44 \pm 0.38$ & $2.57 \pm 0.12$ \\
\hline Liver index & $410 \pm 2$ & $429 \pm 1$ & $437 \pm 1$ & $551 \pm 3$ & $539 \pm 5$ & $555 \pm 2$ \\
\hline
\end{tabular}

Measurements were obtained after 8 weeks (prevention) or 16 weeks (treatment) of administration with either HFD (DIO) or L. laricina at 125 or $250 \mathrm{mg} / \mathrm{kg}$, which was incorporated in the HFD for 8 weeks in the prevention protocol and for the last 8 of 16 weeks in the treatment protocol. The liver index corresponds to liver weight $(\mathrm{mg}) /$ body weight $(\mathrm{mg})$. All values represent the mean $\pm \mathrm{SEM}$ (prevention protocol DIO $=11 ;$ L. laricina $125=13 ; \mathrm{L}$. laricina $250=13$, and for the treatment protocol DIO $=7$; L. laricina $125=5 ;$ L. laricina $250=8$ ). * denotes that treated groups are significantly different as compared to DIO (one-way ANOVA; post hoc analysis Holm-Sidak or Bonferroni-Dunn test; $P<0.05)$.

noncompliance with western medicines could account for the staggering diabetes prevalence of $29 \%$ in adults over 20 years old in this community $[5,6,11-13]$.

In our quest to discover a culturally adapted diabetes treatment, we identified L. laricina from the CEI pharmacopoeia as potential antidiabetic medicinal plant. Initial in vitro screening showed that this plant stimulated glucose uptake, potentiated adipogenesis [24], activated AMPK, and acted as mitochondrial uncoupler/inhibitor (on normal isolated mitochondria) [20]. These effects are reminiscent of the action of Metformin, which partially mediates its action through inhibition of mitochondrial respiration, activation of AMPK, and upregulation of glucose uptake as well [20, 35-39]. It was therefore interesting to test the antidiabetic effect of $L$. laricina in a mouse model mimicking type 2 diabetes as a consequence of obesity by subjecting C57BL/6 mice to HFD (diet-induced-obesity model; DIO). We tested the plant in two different regiments: (1) L. laricina was administered concomitantly with HFD for 8 weeks in order to confirm its capacity to attenuate the development of obesity, diabetes, and the associated insulin-resistance (prevention study); (2) L. laricina was administered to obese and insulin resistant C57BL/6 mice (already on HFD for 8 weeks) for 8 weeks to test its ability to reverse the establishment of both of these states (treatment study).

Typically, the DIO model is characterized by increased body weight gain, hyperglycemia, and establishment of insulin-resistant state (hyperinsulinemia, increase of the leptin/adiponectin ratio, ectopic fat storage in the liver and muscle) $[40,41]$. We therefore examined these parameters to determine the effect of L. laricina in vivo.

The results of the present studies confirm that L. laricina holds great promise as an antidiabetic medicinal plant. Although this plant had no effect of glycemia when administered concomitantly with HFD (prevention study), on the contrary, it significantly and dose-dependently decreased glycemia in the treatment study. These findings correlate well with our in vitro data where this plant extract increased glucose uptake in skeletal muscle cells and adipocytes [24], which accounts for $85 \%$ of postprandial glucose disposal, [42] and increased AMPK activity in C2C12 muscle cells
[20]. It is worthy to note that glycemia levels of animals receiving HFD in the treatment study are higher than those in the prevention study (32\% versus $19 \%$, respectively, compared to CHOW). One could suggest that L. laricina exerts its antihyperglycemic effect better when disease processes are more pronounced, thus explaining the observed difference in the plant's effect between the prevention and the treatment study.

Insulin resistance parameters were also modulated with administration of $L$. laricina in both treatment regiments. While strong tendencies are apparent in the prevention study, insulinemia and leptin/adiponectin ratio were significantly decreased in the treatment study (especially with the highest dose of $L$. laricina), suggesting improvement of systemic insulin resistance. Intriguingly, L. laricina failed to decrease hepatic and muscle triglycerides in both studies. Several lines of evidence suggest that hepatic triglyceride accumulation leads to insulin-defective signaling in the liver with increased hepatic glucose output. However, Buettner et al. have shown that TG accumulation in the liver is not always sufficient to impair insulin signaling $[43,44]$. In fact, they argue that systemic factors (such as adipokines, free fatty acids, proinflammatory cytokines) may play an important role in the regulation of hepatic glucose output and insulin sensitivity in vivo $[43,44]$. Hence, our data on the lack of depletion in intrahepatic and intramuscular triglyceride levels needs to be evaluated in further detail. Indeed, continued administration of HFD alongside L. laricina could make elimination of steatosis difficult. Another possibility could be that since oxidation pathways are saturated with fatty acids being mobilized from the adipose tissue (decrease in adipose tissue weight due to probable hormone-sensitive lipase activity), this could consequently hinder any decrease in tissue triglyceride stores [45]. In all cases, since adiponectin levels tended to increase and leptin/adiponectin ratio (an indicator of insulin resistance) [46-50] significantly decreased with ingestion of L. laricina, proinsulin-resistant systemic factors seem to be decreased and insulin sensitivity improved. Interestingly, we have shown that treatment of hepatic cells in vitro by L. laricina inhibits the activity of enzymes implicated in hepatic gluconeogenesis, such as glucose-6-phosphatase 
TABLE 4: Effects of obesity as well as L. laricina administration on hepatic mitochondrial function.

\begin{tabular}{|c|c|c|c|c|}
\hline & \multicolumn{4}{|c|}{ Treatment protocol } \\
\hline & Chow & DIO & $\begin{array}{l}\text { L. laricina } \\
125 \mathrm{mg} / \mathrm{kg}\end{array}$ & $\begin{array}{l}\text { L. laricina } \\
250 \mathrm{mg} / \mathrm{kg}\end{array}$ \\
\hline State 3 & $62.90 \pm 4.50$ & $59.81 \pm 8.34$ & $51.41 \pm 6.91$ & $62.91 \pm 3.34$ \\
\hline State 4 & $18.23 \pm 1.05$ & $17.43 \pm 0.89$ & $16.20 \pm 1.04$ & $16.29 \pm 0.85$ \\
\hline RCR & $3.45 \pm 0.08$ & $3.37 \pm 0.32$ & $3.18 \pm 0.35$ & $3.87 \pm 0.14$ \\
\hline ATP synthesis & $3.53 \pm 0.53$ & $3.20 \pm 0.52$ & $2.73 \pm 0.40$ & $3.52 \pm 0.25$ \\
\hline
\end{tabular}

Mitochondrial function was measured as described in detail in Section 2.10, after 16 weeks of administration with either standard diet (CHOW), HFD (DIO), or L. laricina at 125 or $250 \mathrm{mg} / \mathrm{kg}$, which was incorporated in the last 8 of 16 weeks in the treatment protocol. State 3 represents the rate of oxygen consumed during oxidative phosphorylation; state 4 represents the rate of oxygen consumption obtained after oxidative phosphorylation; RCR (respiratory control ratio) represents the ratio between state 3 and state 4 . All values represent the mean $\pm \mathrm{SEM}$ (for the treatment protocol CHOW $=4 ; \mathrm{DIO}=5 ; L$. laricina $125=4 ; L$. laricina $250=4)$.

and activates those promoting glycogen formation, such as glycogen synthase (GS), thus directly modulating hepatic glucose output [51].

This plant showed slight decrease of body weight with both studies, which was significant if continuous measurements were taken into account for the first and second month of administration. These changes occurred while the animals were on a continuous hypercaloric/fat-laden diet and without any observed change in energy intake. This could represent an indirect modulation of body weight as a consequence of L. laricina antidiabetic activity, which in some cases is similar to Metformin.

L. laricina administration also decreased retroperitoneal fat pad weight significantly in the prevention study and showed a tendency to do so in the treatment study. This represents an important action in the fight against insulin resistance since visceral adipose tissue has been implicated in the detrimental effects of obesity and insulin resistance [52]. Hence, modulation of this tissue would influence adipokine secretion and contribute to the improvement of insulin sensitivity, as can be seen in our plant-treated mice.

On the molecular level, we have shown that L. laricina activates AMPK in C2C12 myotubes [20] and H4IIE hepatic cell line [51]. This activation may be secondary to a variety of factors, including adiponectin or metabolic stress induced by the disruption of mitochondrial energy transduction [5356]. In the literature, it has been reported that animals (mice or rat) fed a high-fat diet exhibit a decreased mitochondrial respiratory capacity (state 3/state 4 ), as was observed in this treatment protocol in mice administered a HFD [57]. Increased consumption of dietary fat may lead to alterations in mitochondrial membrane composition and increased ROS production and peroxidation of fatty acids, which could damage mitochondrial structures, all affecting mitochondrial function [57]. Uncoupling agents are beneficial in alleviating the mitochondrial stress induced by a HFD, by increasing fatty acid oxidation and decreasing ROS production [58]. In the treatment study, L. laricina at $250 \mathrm{mg} / \mathrm{kg}$ improved mitochondrial capacity and ATP production to levels comparable to those observed in animals fed a standard Chow diet. As demonstrated in previous in vitro screening studies, the uncoupling effect of L. laricina is shortlived and is followed by a prolonged activation of AMPK and an overshoot phenomenon occurring to restore energy homeostasis, where ATP production is greatly increased, through raised carbohydrate and lipid oxidation [20]. Other benefits of increased AMPK activity include protecting cells from further damage by potentiating mitochondrial biogenesis $[20,59,60]$. Therefore, it seems that in the current animal treatment protocol, the long-term effect of L. laricina improved mitochondrial capacity and most probably through AMPK activation regulated glucose homeostasis. Of note, uncoupling agents usually lead to increased heat production due to increased energy expenditure. L. laricinatreated animals in the prevention study exhibited elevated skin temperature, thus confirming its uncoupling activity in vivo.

In conclusion, this study confirms the antidiabetic activity of L. laricina in the context of diet-induced obesity in a mouse model. The results clearly show that $L$. laricina decreased hyperglycemia and insulin resistance and improved mitochondrial function in the treatment study, while partially modulating parameters involved in insulin sensitivity in the prevention one. It also had a slight effect on body weight gain in both studies. The exact mechanisms of action of $L$. laricina remain to be identified, but results point toward possible activation of AMPK and its downstream effectors. In view of the soaring increase in both obesity and diabetes among aboriginal populations and in particular the CEI, L. laricina represents a valuable alternative, and culturally adapted treatment for both these diseases.

\section{Conflict of Interests}

The authors declare no conflict of interests.

\section{Acknowledgments}

A Team Grant from the Canadian Institutes of Health Research (CTP-79855) to P. S. Haddad, L. Musallam, and J. T. Arnason funded these studies. Very special thanks are due to S. Petawabano, L. Petawabano, C. Coon, S. Coon, M. Gunner, A. Mark, K. Mark, E. C. Come, H. Matoush, S. Matoush, R. Coon, and E. C. Come from the Cree Nation of Mistissini and A. Mamianskum, A. Kawapit, A. Natachequan, A. Sandy, E. G. Mamianskum, E. Kawapit, 
J. Kawapit, J. Masty, J. Petagumskum Sr., L. Rupert, M. Natachequan, and M. Natachequan from Whapmagoostui First nation, as well as to 41 other Cree Elders of both nations who kindly agreed to be interviewed. They made this paper possible by allowing the authors to use, for the purposes of this research, their knowledge relating to medicinal plants transmitted to them by their elders. Their trust has also enabled a useful exchange between indigenous knowledge and Western science.

\section{References}

[1] PHAC, Obesity in Canada-Snapshot, 2009.

[2] PHAC, Obesity in Canada: A Joint Report from the Public Health Agency of Canada and the Canadian Institute for Health Information, 2011.

[3] PHAC, "Diabetes among first Nations, inuit, and Métis populations," in Diabetes in Canada: Facts and Figures from a Public Health Perspective, chapter 6, 2011.

[4] Statcan, "Diabetes," in Statistics Canada, 2010.

[5] D. Dannenbaum, E. Kuzmina, P. Lejeune, J. Torrie, and M. Gangbe, "Prevalence of diabetes and diabetes-related complications in first nations communities in Northern Quebec (Eeyou Istchee), Canada," Canadian Journal of Diabetes, vol. 32, no. 1, pp. 46-52, 2008.

[6] E. Kuzmina, P. Lejeune, D. Dannenbaum, and J. E. Torrie, "Cree diabetes information system (CDIS)," in Annual Report, J. E. Torrie, Ed., 2009.

[7] P. Brassard, E. Robinson, and C. Lavallee, "Prevalence of diabetes mellitus among the James Bay Cree of northern Quebec," Canadian Medical Association Journal, vol. 149, no. 3, pp. 303-307, 1993.

[8] E. Kuzmina and D. Dannenbaum, "Cree diabetes information system (CDIS)," in Annual Report, 2004.

[9] G. Légaré, Projet de Surveillance du Diabète Chez les Cris d'Eeyou Istchee, Institut National de Santé Publique du Québec et Conseil Cri de la Santé et des Services Sociaux de la BaieJames, Quebec, Canada, 2004.

[10] G. Légaré, D. Dannenbaum, J. Torrie, E. Kuzmina, and P. Linton, Effects of Diabetes on the Health of the Cree of Eeyou Istchee: What Can be Learned from Linking the Cree Diabetes Information System (CDIS) with the Quebec Diabetes Surveillance System (QDSS), Public Health Occasional, 2004, Edited by Bay IndspdQaCBoHaSSoJ.

[11] P. Boston, S. Jordan, E. MacNamara et al., "Using participatory action research to understand the meanings aboriginal canadians attribute to the rising incidence of diabetes," Chronic Diseases in Canada, vol. 18, no. 1, pp. 5-12, 1997.

[12] R. A. Hegele, "Genes and environment in type 2 diabetes and atherosclerosis in aboriginal Canadians," Current atherosclerosis reports, vol. 3, no. 3, pp. 216-221, 2001.

[13] T. K. Young, J. Reading, B. Elias, and J. D. O’Neil, “Type 2 diabetes mellitus in Canada's first nations: status of an epidemic in progress," Canadian Medical Association Journal, vol. 163, no. 9, pp. 561-566, 2000.

[14] K. Gray-Donald, E. Robinson, A. Collier, K. David, L. Renaud, and S. Rodrigues, "Intervening to reduce weight gain in pregnancy and gestational diabetes mellitus in Cree communities: an evaluation," Canadian Medical Association Journal, vol. 163, no. 10, pp. 1247-1251, 2000.

[15] C. Leduc, J. Coonishish, P. Haddad, and A. Cuerrier, "Plants used by the cree nation of eeyou istchee (Quebec, Canada) for the treatment of diabetes: a novel approach in quantitative ethnobotany," Journal of Ethnopharmacology, vol. 105, no. 1-2, pp. 55-63, 2006.

[16] H. M. Eid, L. C. Martineau, A. Saleem et al., "Stimulation of AMP-activated protein kinase and enhancement of basal glucose uptake in muscle cells by quercetin and quercetin glycosides, active principles of the antidiabetic medicinal plant vaccinium vitis-idaea," Molecular Nutrition and Food Research, vol. 54, no. 7, pp. 991-1003, 2010.

[17] H. M. Eid, D. Vallerand, A. Muhammad, T. Durst, P. S. Haddad, and L. C. Martineau, "Structural constraints and the importance of lipophilicity for the mitochondrial uncoupling activity of naturally occurring caffeic acid esters with potential for the treatment of insulin resistance," Biochemical Pharmacology, vol. 79, no. 3, pp. 444-454, 2010.

[18] M. H. Fraser, A. Cuerrier, P. S. Haddad, J. T. Arnason, P. L. Owen, and T. Johns, "Medicinal plants of Cree communities (Québec, Canada): antioxidant activity of plants used to treat type 2 diabetes symptoms," Canadian Journal of Physiology and Pharmacology, vol. 85, no. 11, pp. 1200-1214, 2007.

[19] D. Harbilas, L. C. Martineau, C. S. Harris et al., "Evaluation of the antidiabetic potential of selected medicinal plant extracts from the Canadian boreal forest used to treat symptoms of diabetes: part II," Canadian Journal of Physiology and Pharmacology, vol. 87, no. 6, pp. 479-492, 2009.

[20] L. C. Martineau, D. C. A. Adeyiwola-Spoor, D. Vallerand, A. Afshar, J. T. Arnason, and P. S. Haddad, "Enhancement of muscle cell glucose uptake by medicinal plant species of Canada's native populations is mediated by a common, Metformin-like mechanism," Journal of Ethnopharmacology, vol. 127, no. 2, pp. 396-406, 2010.

[21] L. C. Martineau, J. Hervé, A. Muhamad et al., "Anti-adipogenic activities of Alnus incana and Populus balsamifera bark extracts, part I: sites and mechanisms of action," Planta Medica, vol. 76, no. 13, pp. 1439-1446, 2010.

[22] L. C. Martineau, A. Muhammad, A. Saleem et al., "Anti-adipogenic activities of alnus incana and populus balsamifera bark extracts, part II: bioassay-guided identification of actives salicortin and oregonin," Planta Medica, vol. 76, no. 14, pp. 1519-1524, 2010.

[23] L. A. Nistor Baldea, L. C. Martineau, A. BenhaddouAndaloussi, J. T. Arnason, E. Levy, and P. S. Haddad, "Inhibition of intestinal glucose absorption by anti-diabetic medicinal plants derived from the James Bay Cree traditional pharmacopeia," Journal of Ethnopharmacology, vol. 132, no. 2, pp. 473-482, 2010.

[24] D. C. A. Spoor, L. C. Martineau, C. Leduc et al., "Selected plant species from the Cree pharmacopoeia of northern Quebec possess anti-diabetic potential," Canadian Journal of Physiology and Pharmacology, vol. 84, no. 8-9, pp. 847-858, 2006.

[25] M. S. Eberhardt, C. Ogden, M. Engelgau, B. Cadwell, A. A. Hedley, and S. H. Saydah, Prevalence of Overweight and Obesity Among Adults with Diagnosed Diabetes, CDC, and Department of Health and Human Services, 2004.

[26] A. R. Desilets, S. Dhakal-Karki, and K. C. Dunican, "Role of metformin for weight management in patients without type 2 diabetes," The Annals of Pharmacotherapy, vol. 42, no. 6, pp. 817-826, 2008.

[27] S. M. Grundy, "Drug therapy of the metabolic syndrome: minimizing the emerging crisis in polypharmacy," Nature Reviews Drug Discovery, vol. 5, no. 4, pp. 295-309, 2006.

[28] M. L. Peyot, E. Pepin, J. Lamontagne et al., “ $\beta$-cell failure in diet-induced obese mice stratified according to body weight gain: secretory dysfunction and altered islet lipid metabolism 
without steatosis or reduced $\beta$-cell mass," Diabetes, vol. 59, no. 9, pp. 2178-2187, 2010.

[29] J. Folch, M. Lees, and G. H. Sloane Stanley, "A simple method for the isolation and purification of total lipides from animal tissues," The Journal of Biological Chemistry, vol. 226, no. 1, pp. 497-509, 1957.

[30] R. Dallmann, S. Steinlechner, S. von Horsten, and T. Karl, "Stress-induced hyperthermia in the rat: comparison of classical and novel recording methods," Laboratory Animals, vol. 40, no. 2, pp. 186-193, 2006.

[31] A. Benhaddou-Andaloussi, L. C. Martineau, D. Vallerand et al., "Multiple molecular targets underlie the antidiabetic effect of Nigella sativa seed extract in skeletal muscle, adipocyte and liver cells," Diabetes, Obesity and Metabolism, vol. 12, no. 2, pp. 148-157, 2009.

[32] D. Johnson and H. Lardy, "Isolation of liver or kidney mitochondria," Methods in Enzymology, vol. 10, pp. 94-96, 1967.

[33] H. Ligeret, A. Brault, D. Vallerand, Y. Haddad, and P. S. Haddad, "Antioxidant and mitochondrial protective effects of silibinin in cold preservation-warm reperfusion liver injury," Journal of Ethnopharmacology, vol. 115, no. 3, pp. 507-514, 2008.

[34] IDF, One Adult in Ten has Diabetes in North America, 51.2 Million People in North America and the Caribbean will be Living with the Disease by 2030, International Diabetes Federation, Brussels, Belgium, 2011, Edited by Federation I. D., World Diabetes Day.

[35] B. Brunmair, K. Staniek, F. Gras et al., "Thiazolidinediones, like metformin, inhibit respiratory complex I: a common mechanism contributing to their antidiabetic actions?" Diabetes, vol. 53, no. 4, pp. 1052-1059, 2004.

[36] E. Elia, V. Sander, C. G. Luchetti et al., "The mechanisms involved in the action of metformin in regulating ovarian function in hyperandrogenized mice," Molecular Human Reproduction, vol. 12, no. 8, pp. 475-481, 2006.

[37] Y. D. Kim, K. G. Park, Y. S. Lee et al., "Metformin inhibits hepatic gluconeogenesis through AMP-activated protein kinasedependent regulation of the orphan nuclear receptor SHP," Diabetes, vol. 57, no. 2, pp. 306-314, 2008.

[38] J. M. Lee, W. Y. Seo, K. H. Song et al., "AMPK-dependent repression of hepatic gluconeogenesis via disruption of CREB.CRTC2 complex by orphan nuclear receptor small heterodimer partner," The Journal of Biological Chemistry, vol. 285, no. 42, pp. 32182-32191, 2010.

[39] B. Viollet, F. Andreelli, S. B. Jorgensen et al., "Physiological role of AMP-activated protein kinase (AMPK): insights from knockout mouse models," Biochemical Society Transactions, vol. 31, no. 1, pp. 216-219, 2003.

[40] W. T. Cefalu, "Animal models of type 2 diabetes: clinical presentation and pathophysiological relevance to the human condition," ILAR News, vol. 47, no. 3, pp. 186-198, 2006.

[41] T. Jiang, Z. Wang, G. Proctor et al., "Diet-induced obesity in $\mathrm{C} 57 \mathrm{BL} / 6 \mathrm{~J}$ mice causes increased renal lipid accumulation and glomerulosclerosis via a sterol regulatory element-binding protein-1c-dependent pathway," The Journal of Biological Chemistry, vol. 280, no. 37, pp. 32317-32325, 2005.

[42] R. A. DeFronzo, "Pathogenesis of type 2 diabetes mellitus," The Medical Clinics of North America, vol. 88, no. 4, pp. 787$835,2004$.

[43] R. Buettner, I. Ottinger, J. Scholmerich, and L. C. Bollheimer, "Preserved direct hepatic insulin action in rats with dietinduced hepatic steatosis," American Journal of Physiology. Endocrinology and Metabolism, vol. 286, no. 5, pp. E828-E833, 2004.
[44] R. Buettner, J. Schölmerich, and L. C. Bollheimer, "High-fat diets: modeling the metabolic disorders of human obesity in rodents," Obesity, vol. 15, no. 4, pp. 798-808, 2007.

[45] R. Crescenzo, F. Bianco, I. Falcone et al., "Hepatic mitochondrial energetics during catch-up fat with high-fat diets rich in lard or safflower oil," Obesity. In press.

[46] F. Abbasi, J. W. Chu, C. Lamendola et al., "Discrimination between obesity and insulin resistance in the relationship with adiponectin," Diabetes, vol. 53, no. 3, pp. 585-590, 2004.

[47] F. M. Finucane, J. Luan, N. J. Wareham et al., "Correlation of the leptin: adiponectin ratio with measures of insulin resistance in non-diabetic individuals," Diabetologia, vol. 52, no. 11, pp. 2345-2349, 2009.

[48] O. Tschritter, A. Fritsche, C. Thamer et al., "Plasma adiponectin concentrations predict insulin sensitivity of both glucose and lipid metabolism," Diabetes, vol. 52, no. 2, pp. 239243, 2003.

[49] C. Weyer, T. Funahashi, S. Tanaka et al., "Hypoadiponectinemia in obesity and type 2 diabetes: close association with insulin resistance and hyperinsulinemia," Journal of Clinical Endocrinology and Metabolism, vol. 86, no. 5, pp. 1930-1935, 2001.

[50] Y. Yamamoto, H. Hirose, I. Saito et al., "Correlation of the adipocyte-derived protein adiponectin with insulin resistance index and serum high-density lipoprotein-cholesterol, independent of body mass index, in the Japanese population," Clinical Science, vol. 103, no. 2, pp. 137-142, 2002.

[51] A. Nachar, A. Saleem, D. Vallerand et al., "Beneficial effects in the liver of antidiabetic plants used in traditional medicine by the Cree of Bay James in Canada," in Proceedings of the 10th Annual Oxford International Conference on the Science of Botanicals, Planta Medica, Mississipi, Miss, USA, 2011.

[52] E. S. Freedland, "Role of a critical visceral adipose tissue threshold (CVATT) in metabolic syndrome: Implications for controlling dietary carbohydrates: a review," Nutrition and Metabolism, vol. 1, no. 1, article 12, 2004.

[53] Z. P. Chen, K. I. Mitchelhill, B. J. Michell et al., "AMP-activated protein kinase phosphorylation of endothelial NO synthase," FEBS Letters, vol. 443, no. 3, pp. 285-289, 1999.

[54] N. Fujii, N. Jessen, L. J. Goodyear et al., "AMP-activated protein kinase and the regulation of glucose transport," American Journal of Physiology. Endocrinology and Metabolism, vol. 291, no. 5, pp. E867-E877, 2006.

[55] N. Ruderman and M. Prentki, "AMP kinase and malonylCoA: targets for therapy of the metabolic syndrome," Nature Reviews. Drug Discovery, vol. 3, no. 4, pp. 340-351, 2004.

[56] W. W. Winder and D. G. Hardie, "AMP-activated protein kinase, a metabolic master switch: possible roles in type 2 diabetes," The American Journal of Physiology, vol. 277, no. 1, part 1, pp. E1-E10, 1999.

[57] C. Raffaella, B. Francesca, F. Italia, P. Marina, L. Giovanna, and I. Susanna, "Alterations in hepatic mitochondrial compartment in a model of obesity and insulin resistance," Obesity, vol. 16, no. 5, pp. 958-964, 2008.

[58] M. K. C. Hesselink, M. Mensink, and P. Schrauwen, "Lipotoxicity and mitochondrial dysfunction in type 2 diabetes," Immunology, Endocrine \& Metabolic Agents in Medicinal Chemistry, vol. 7, pp. 3-17, 2007.

[59] R. M. Reznick and G. I. Shulman, "The role of AMP-activated protein kinase in mitochondrial biogenesis," The Journal of Physiology, vol. 574, part 1, pp. 33-39, 2006.

[60] W. W. Winder, "Energy-sensing and signaling by AMP-activated protein kinase in skeletal muscle," Journal of Applied Physiology, vol. 91, no. 3, pp. 1017-1028, 2001. 


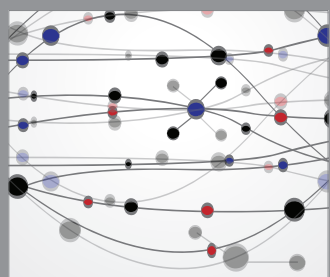

The Scientific World Journal
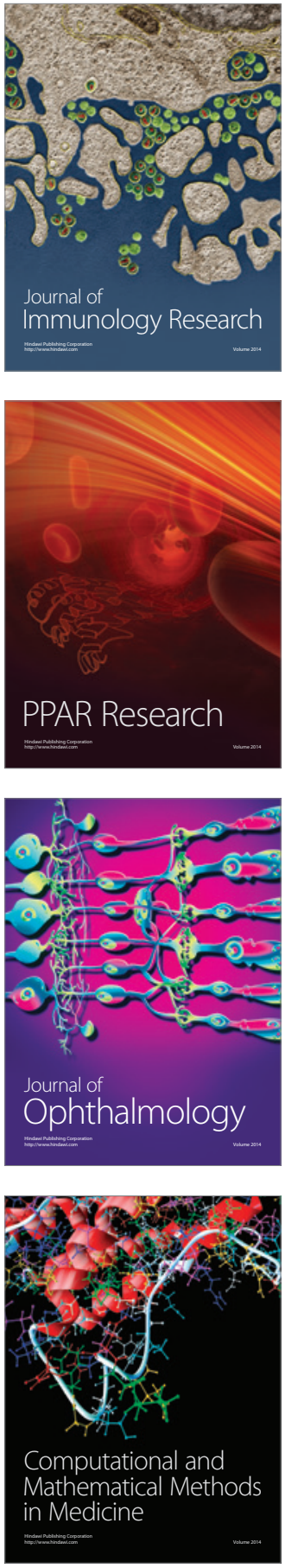

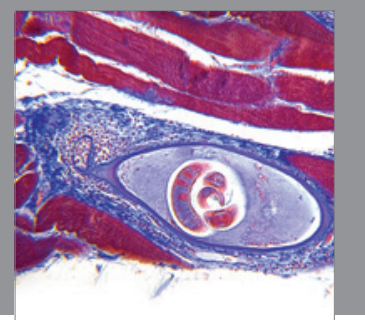

Gastroenterology

Research and Practice
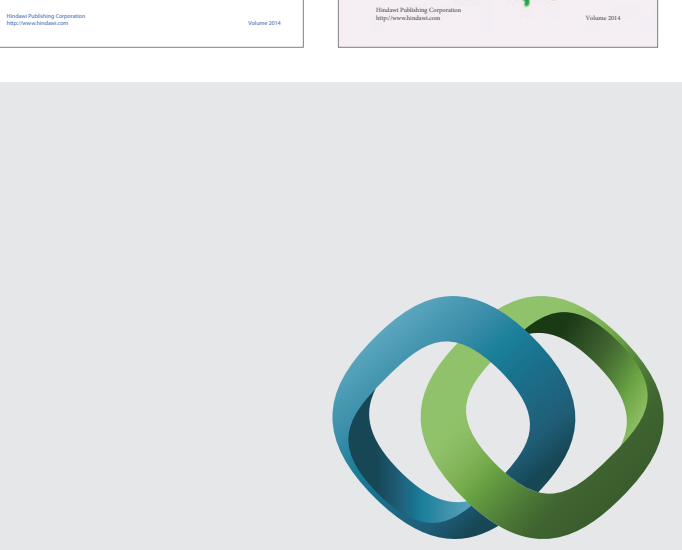

\section{Hindawi}

Submit your manuscripts at

http://www.hindawi.com
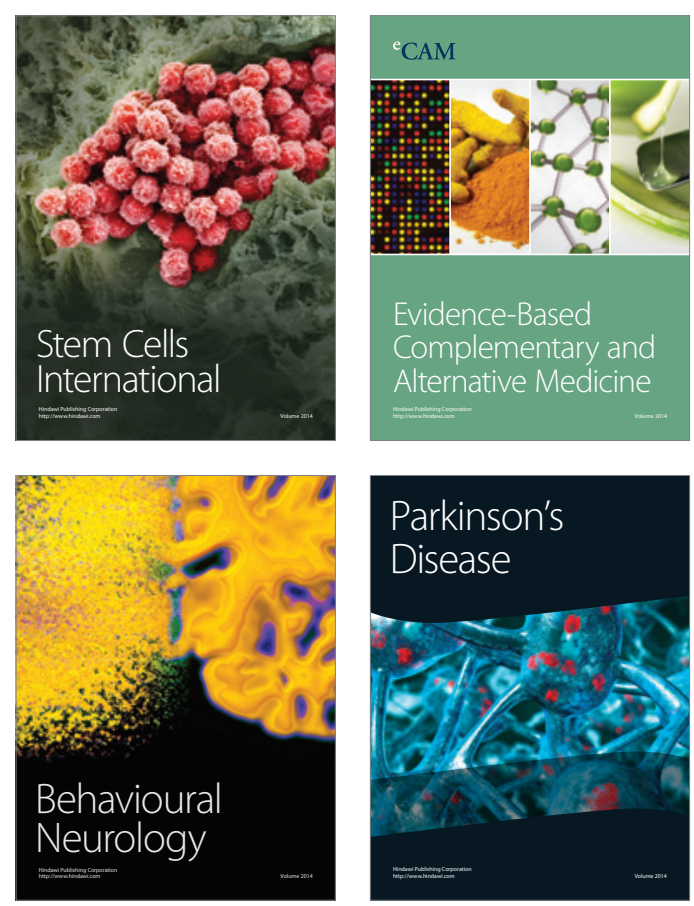

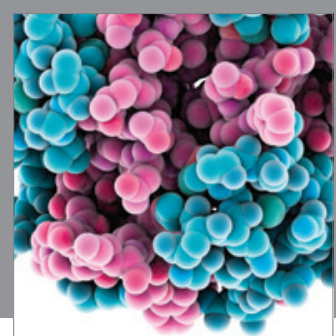

Journal of
Diabetes Research

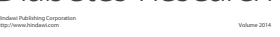

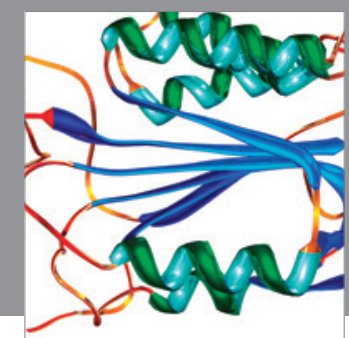

Disease Markers
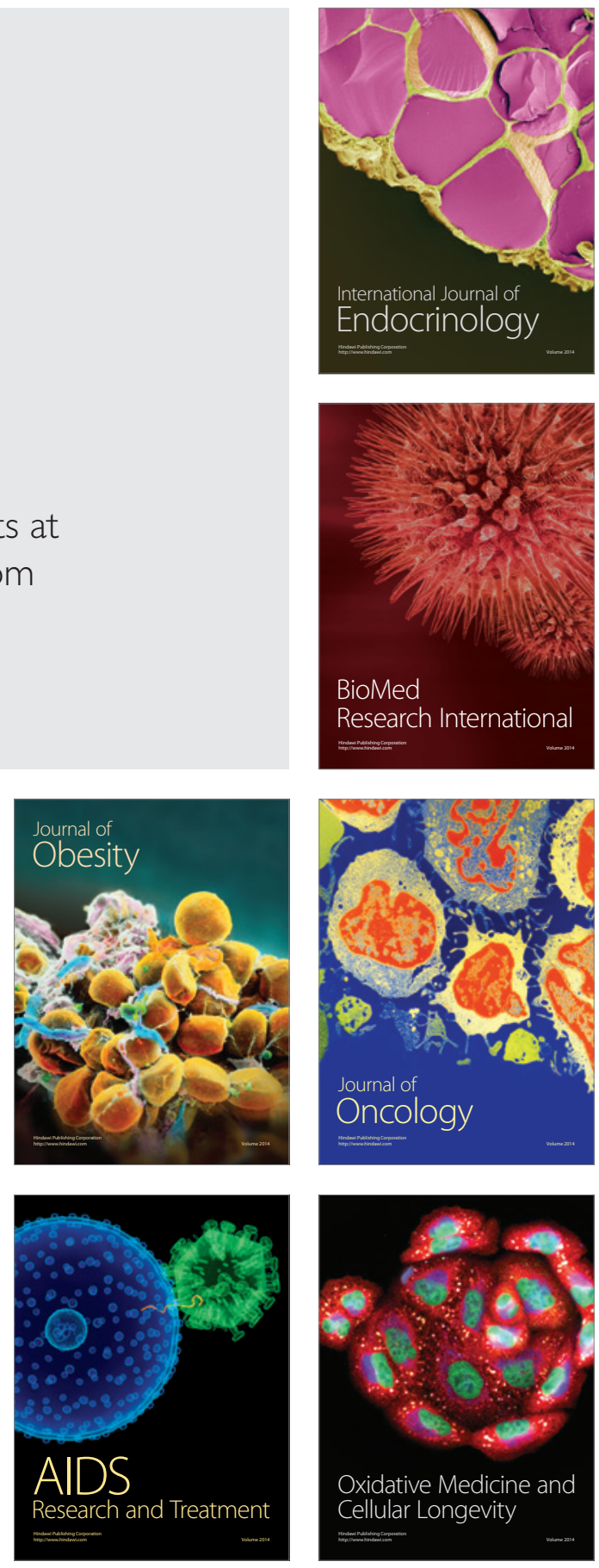